\title{
Investigando Curadoria Coletiva em um Contexto Científico de Compartilhamento de Métodos e Modelos
}

\author{
Lídia Silva Ferreira \\ Universidade Federal de Minas Gerais \\ Belo Horizonte, Minas Gerais \\ lidiaferreira@dcc.ufmg.br
}

\author{
Raquel Oliveira Prates \\ Universidade Federal de Minas Gerais \\ Belo Horizonte, Minas Gerais \\ rprates@dcc.ufmg.br
}

\begin{abstract}
RESUMO
Este trabalho tem por objetivo investigar estratégias de curadoria coletiva no contexto científico, ou seja, analisar como as pessoas colaboram entre si para fazer a seleção de um determinado tipo de informação e como um sistema pode intermediar essa interação. Uma vez que tais estratégias sejam identificadas, deseja-se fazer um estudo exploratório para avaliar tanto a estratégia quanto a sua aplicação por um sistema. Para isso, espera-se implementar as estratégias de curadoria em um estudo de caso no contexto de métodos de avaliação de sistemas usando o StoreAnt, um repositório de caracterização de métodos, como base para aplicação e validação das estratégias de curadoria investigadas. Isso provê como resultado contribuições teóricas e práticas. Como contribuições teóricas tem-se principalmente os resultados da investigação sobre estratégias de curadoria coletiva, de forma consolidada, além de critérios de busca, caracterização e comparação em um contexto específico de métodos de avaliação. Hoje esse é um tema pouco explorado na área de IHC e com grande potencial para a comunidade, uma vez que impacta diretamente nos mecanismos de consolidação dos trabalhos publicados. Como contribuição prática, tem-se os resultados do estudo de caso e a consolidação da ferramenta StoreAnt, que poderá ser utilizada pela comunidade para intermediar a curadoria de um determinado tipo de conhecimento.
\end{abstract}

\section{PALAVRAS-CHAVE}

curadoria coletiva, métodos de avaliação de sistemas, sistemas colaborativos

\footnotetext{
Permission to reproduce or distribute, in whole or in part, material extracted from this work, verbatim, adapted or remixed, as well as the creation or production from the content of such work, is granted without fee for noncommercial use, provided that the original work is properly credited.

IHC 2019 - WORKSHOP DE TESES E DISSERTAÇÕES, Outubro 21-25, 2019, Vitória, Brasil. In Anais Estendidos do XVIII Simpósio Brasileiro sobre Fatores Humanos em Sistemas Computacionais. Porto Alegre: SBC.

(C) 2019 by the author(s), in accordance with the terms of the Creative Commons Attribution-NonCommercial 4.0 International Public License (CC BY-NC 4.0).
}

\section{INTRODUÇÃO}

Curadoria é uma prática comum na Arqueologia. Nesta ciência, tal atividade consiste em coletar, avaliar, organizar e preservar um conjunto de recursos para uso futuro [Bamforth 1986]. Em várias plataformas online o mesmo conceito é aplicado e chamado de "Curadoria Digital"1, que consiste em manter, preservar e agregar valor a dados digitais, muitas vezes através de sua disponibilização em bibliotecas e repositórios virtuais [Wu et al. 2017]. Esses materiais podem ser dos mais diferentes tipos, organizados de diferentes formas, por diferentes grupos. Podem ser páginas, fotos, avaliação de serviços, tweets, arquivos de áudio, entre outros.

Um problema presente na área de IHC está relacionado à curadoria dos métodos, das ferramentas conceituais e dos modelos publicados em conferências. A publicação de um novo modelo ou método não é garantia de sua consolidação, mesmo que já tenham sido avaliado. Para isso, é necessário que os artefatos sejam usados, testados e avaliados por um determinado período de tempo, idealmente por diferentes pessoas e em diferentes contextos. Como permitir que as pessoas disponibilizem, avaliem, critiquem e gerem informações sobre esses métodos e modelos? Como permitir que as pessoas de uma comunidade façam a curadoria deles, para que se beneficiem do conhecimento coletivo sobre uma ferramenta publicada? Para responder a essa e a outras questões, foi proposto esse trabalho.

\section{TRABALHOS RELACIONADOS}

Foram encontrados e analisados, inicialmente, quatro artigos relacionados ao tema da curadoria coletiva.

Um dos mais relevantes para este estudo, o trabalho de [Wu et al. 2017] mostra como foi feita a apropriação do GitHub para fins de curadoria coletiva. Os autores investigaram o que motiva os desenvolvedores a fazer a curadoria, porque eles usam o GitHub, como o conteúdo curado é utilizado pelo público e explora como a plataforma pode apoiar ainda mais esse processo. São levantadas também as motivações da comunidade para curar esse conhecimento, através de uma entrevistas feitas com 16 curadores. O trabalho de

\footnotetext{
${ }^{1}$ Digital Curation Centre (DCC) - http://www.dcc.ac.uk/about-us
} 
[Wu et al. 2018] vai na mesma linha, indo mais a fundo na investigação dos recursos do GitHub que são explorados pelos curadores, das características do conteúdo e dos padrões de colaboração nos repositórios de conteúdo curado. Nesse trabalho, foram feitas analises de logs e conteúdo de repositórios populares. $\mathrm{O}$ estudo mostrou que a curadoria pode ser uma forma importante de disseminação de conhecimento pelos desenvolvedores, que o papel de curador pode se tornar importante na comunidade e que eles podem usar da curadoria do conhecimento para se fazerem notar.

O trabalho de [Zagalsky et al. 2016] mostra um cenário mais específico, explorando como a comunidade de pessoas que utilizam a linguagem $\mathrm{R}$ compartilham e fazem a curadoria do conhecimento que geram. Eles investigam quais os artefatos de conhecimento são compartilhados, como o conhecimento é construído e o que motiva essa interação. Eles apontam em seus resultados duas formas de fazer curadoria: a curadoria participativa, onde vários membros constroem juntos um conhecimento; e a curadoria por crowsorced, onde pessoas trabalham de forma independente.

Por fim, [Voyloshnikova and Storey 2014] avaliaram o potencial de 20 aplicações para uma possível curadoria de informação. Os sistemas não foram usados para curadoria, mas foi feita uma análise do potencial de uso através da aplicação de um framework proposto pelos autores. Foram levantadas quatro principais abordagens de curadoria, os fatores e as questões importantes para a construção e a avaliação de ferramentas Web para a descoberta de informação.

\section{StoreAnt}

O StoreAnt ${ }^{2}$ [Ferreira and Prates 2013][de Souza et al. 2015] é um repositório virtual para catalogação e caracterização de métodos de avaliação de sistemas colaborativos. Ele foi proposto em 2013, como projeto de Iniciação Científica no $\mathrm{PENSi}^{3}$. Para sua concepção, foram usados os critérios de classificação definidos por [Santos et al. 2012] para catalogar métodos de avaliação. O desenvolvimento técnico do StoreAnt, baseado na especificação inicial, começou em 2015. Esse trabalho foi apresentado em uma demo [de Souza et al. 2015] no INTERACT $2015^{4}$.

O sistema encontra-se hoje em fase de desenvolvimento e ainda não foi testado nem avaliado. Espera-se fazê-lo como parte do desenvolvimento deste trabalho de mestrado (ver Seção 4). Mais do que isso, espera-se usar o StoreAnt como ferramenta para o estudo de caso deste trabalho, usando-o para testar hipóteses sobre as estratégias de curadoria coletiva que venham a ser encontradas ou propostas.

\footnotetext{
${ }^{2}$ Acesso em http://storeant.herokuapp.com/.

${ }^{3}$ PENSi: Núcleo de Pesquisa em Engenharia Semiótica e Interação (http://pensi.dcc.ufmg.br/)

${ }^{4}$ International Conference on Human-Computer Interaction (http://www.interact2015.org/)
}

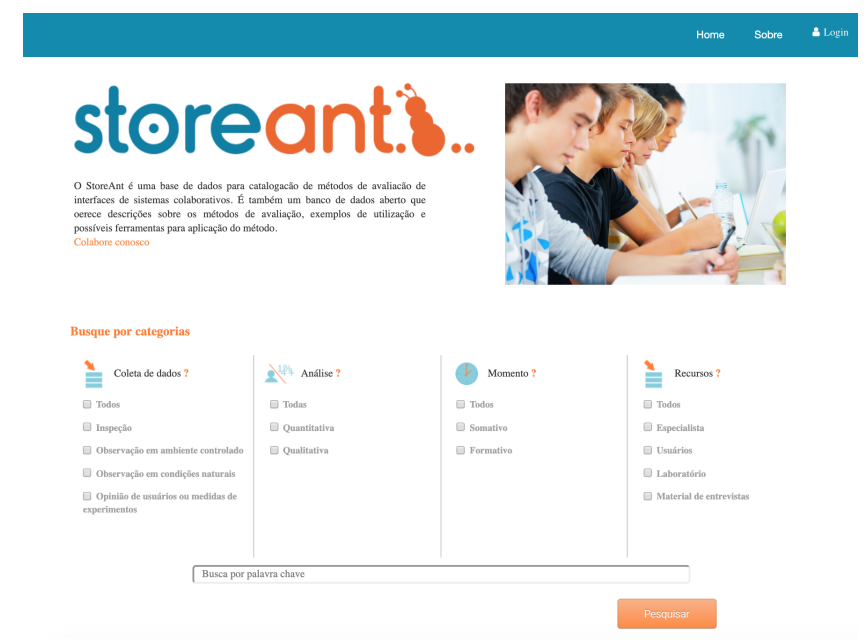

Figura 1: StoreAnt: página inicial com critérios de busca

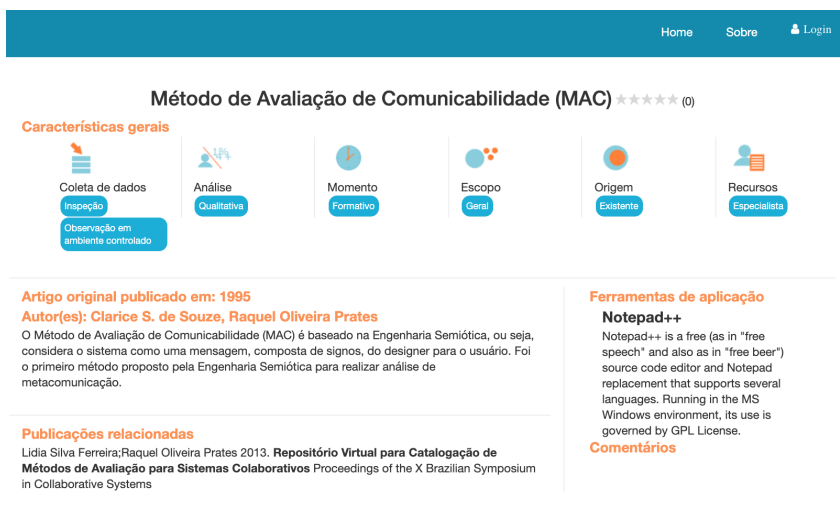

Figura 2: StoreAnt: tela de descrição do método de avaliação

\section{OBJETIVOS}

A proposta do StoreAnt como um repositório virtual surgiu da necessidade de catalogar métodos de avaliação para sistemas colaborativos na comunidade de IHC. Primeiramente, foi proposta uma maneira de catalogar os métodos existentes e suas características, para que o potencial avaliador pudesse identificar, visualizar e futuramente comparar diferentes métodos de avaliação para sistemas colaborativos e apoiar sua escolha nesta ferramenta. Porém, mais do que isso, é necessário também propor uma forma de categorizar e apresentar os dados; por isso a necessidade de se estudar como fazer a curadoria coletiva do que está sendo disponibilizado. Fazer uma curadoria individual de uma determinada área do conhecimento pode ser pouco viável: além de ser um trabalho árduo para a pessoa responsável em fazer a curadoria (que deverá pesquisar, divulgar e acompanhar as aplicações dos artefatos) é difícil de escalar e manter por longos períodos 
de tempo, uma vez que um artefato pode gastar anos até se consolidar.

Dada a necessidade de organizar esse conhecimento, afim de possibilitar a sua consolidação, surge a questão: como fazer a curadoria de tais artefatos de maneira coletiva? Além disso, como garantir que essa curadoria será feita usando os conhecimentos específicos das pessoas envolvidas na curadoria (assim como a seleção de artigos acadêmicos em conferências)? A curadoria de um conhecimento técnico específico feita por pessoas não técnicas, por exemplo, não teria valor, uma vez que dificilmente teríamos massa crítica suficiente para garantir a consolidação do dado. Assim, é necessária a participação da própria comunidade nesse trabalho, caracterizando o processo coletivo. Uma vez que seja possível efetivamente fazer a curadoria dos métodos, ferramentas e modelos propostos em um determinada área do conhecimento, levando em consideração o conhecimento científico associado das pessoas responsáveis pela curadoria nessa avaliação, espera-se possibilitar o uso e a consolidação deste conhecimento de forma mais efetiva. Essa diferença entre o contexto científico e genérico justifica a necessidade de uma investigação de estratégias específicas de curadoria que abranjam as questões apresentadas.

Assim sendo, são objetivos deste trabalho:

(1) Investigar estratégias de curadoria coletiva: como os sistemas e as pessoas fazem esse tipo de seleção de informação.

(2) Identificar aspectos e critérios relevantes para se buscar, comparar e avaliar os métodos de avaliação existentes.

(3) Implementar e testar estratégias de curadoria coletiva em um estudo de caso em um contexto específico e aplicar essas técnicas usando o StoreAnt.

Inicialmente, espera-se focar no estudo das estratégias de curadoria coletiva para a área de IHC, especificamente para catalogação de métodos de avaliação. A longo prazo, esperase investigar a possibilidade de explorar um contexto mais amplo (i.e. gerador de repositórios de conhecimento), não sendo esse o objetivo nesse momento.

\section{METODOLOGIA}

\section{Investigação das estratégias}

Primeiramente é necessário investigar como é feita curadoria em contextos coletivos hoje. Para isso, serão usados dois métodos: uma Revisão Sistemática de Literatura (SLR) e avaliações com o Método de Inspeção Semiótica (MIS).

A SLR [Kitchenham 2004] é um método qualitativo sistemático de pesquisa, que ajuda a explorar as razões pelas quais os fenômenos acontecem, indo além da contagem de sua porcentagem de ocorrência. $\mathrm{O}$ método foi escolhido dado que ele atende ao objetivo da presente pesquisa: interpretar o conteúdo dos artigos retornados, de modo a traçar relações de significado entre eles, contribuindo para a definição das estratégias utilizadas pelos autores dos trabalhos analisados em suas pesquisas. Foram traçadas as seguintes questões de pesquisa:

\section{- Questão 1}

O que tem sido feito, em termos de curadoria de conteúdos relacionados a ciência da computação, nos últimos cinco anos?

- Quais estratégias de curadoria utilizam sistemas digitais, e quais as características desses sistemas?

- Como as pessoas se organizam coletivamente, para a realização dessa curadoria, e como interagem entre si e com o(s) sistema(s) em questão?

- Quais as motivações das pessoas para a realização e uso da curadoria desses conhecimentos?

\section{- Questão 2}

Existe alguma estratégia voltada para a curadoria de métodos de avaliação em geral? E de métodos de avaliação de sistemas digitais?

Uma vez definido o protocolo, o mesmo foi aplicado sobre uma ampla base de dados científicos, envolvendo os principais repositórios acadêmicos da área. A aplicação do protocolo, em sua maior parte, se deu através da execução de strings de busca automática sobre os mesmos.

O MIS [de Souza et al. 2006] é um método antecipativo, ou seja, um especialista inspeciona o sistema em busca de potenciais rupturas de comunicação que poderiam surgir na interação entre o usuário e o sistema. É um método de análise sistemático que vem sendo utilizado na condução de pesquisas científicas, tendo inclusive uma abordagem para esse fim [de Souza and Leitão 2009]. Ele foi escolhido por possibilitar que sistemas em estado formativo ou somativo sejam avaliados e que as estratégias de mediação a curadoria coletiva sejam coletadas.

Assim sendo, será possível entender o que se tem consolidado hoje no contexto de curadoria coletiva através de duas abordagens: analisando os trabalhos relacionados e os sistemas em fase de design ou já em funcionamento que intermediam a interação de interesse.

\section{Análise e proposta de estratégias}

Uma vez que foram identificadas as estratégias existentes, é hora de analisar as que seriam aplicáveis para o contexto específico que se deseja propor ou mesmo propor uma que pareça mais adequada.

É esperado que em uma curadoria coletiva de métodos de avaliação em IHC, por exemplo, tenha-se pessoas com conhecimento específico em métodos de avaliação, para contrapor a falta de uma massa crítica de usuários. Nesse sentido, algumas questões levantadas foram: (1) Como definir que 
a pessoa é especialista em uma área do conhecimento?; (2) Como distribuir os artigos para as pessoas avaliarem sem criar gargalos?; e (3) Como efetivamente não depender de uma massa crítica para garantir a consolidação do dado? Além disso, espera-se propor indicadores de qualidade, que ajudem no controle da qualidade de aspectos como: (1) o nível de conhecimento das pessoas que fazem a curadoria; (2) o material que é curado; e (3) o processo de curadoria em si, intermediado pelo sistema.

\section{Aplicação e avaliação das estratégias propostas}

Uma vez que foram definidas uma ou mais estratégias, desejase aplicá-las em um contexto real de curadoria coletiva de avaliação de métodos de IHC. Esse contexto foi escolhido por ser de domínio dos pesquisadores, possibilitando uma curadorial real dos métodos para avaliação da técnica. Para isso, é necessário implementar a estratégia no sistema StoreAnt, popular o sistema com os métodos de avaliação da literatura e simular o contexto de curadoria real.

Espera-se avaliar o sistema e a estragégia de curadoria inicialmente em um grupo controlado (e.g. os integrantes do PENSi). Posteriormente, espera-se fazer uma avaliação da comunidade de IHC em geral, para que seja possível analisar a aplicabilidade da estratégia de curadoria implementada às necessidades da comunidade. Por fim, espera-se analisar a experiência dessas pessoas com o sistema como um todo, através da coleta e análise de opinião (e.g. entrevistas, questionários e grupos focais).

\section{CONTRIBUIÇÕES ESPERADAS}

Este trabalho prevê dois tipos de contribuições como resultado: contribuições teóricas e contribuições práticas. Como contribuições teóricas tem-se principalmente os resultados da investigação sobre estratégias de curadoria coletiva, de forma consolidada, além de critérios de busca, caracterização e comparação em um contexto específico de métodos de avaliação. Hoje esse é um tema pouco explorado na área de IHC e com grande potencial para a comunidade, uma vez que impacta diretamente nos mecanismos de consolidação dos trabalhos publicados.

Como contribuição prática, tem-se os resultados do estudo de caso e a consolidação da ferramenta StoreAnt. Um exemplo prático pode ser visto na área de avaliação de sistemas colaborativos: avaliar sistemas colaborativos hoje é uma tarefa não trivial em todas as suas etapas [Prates and Raposo 2006]. Até mesmo a escolha do melhor método a ser usado em uma situação/contexto tem sido um desafio. Muitos métodos foram propostos, no entanto poucos são consolidados. Obter conhecimento sobre eles envolve buscas complexas, que dificultam todo o processo. Assim, se propõs a criação do repositório online dos métodos propostos, organizados através da curadoria coletiva. Espera-se que essa ferramenta seja disponibilizada para a comunidade, como forma de aumentar o acesso e o compartilhamento de informações entre seus membros, colaborando para a consolidação dos trabalhos da área e seu fortalecimento, podendo ser útil tanto no contexto acadêmico quanto profissional.

Como trabalho futuro, cabe a discussão dessa aplicação em um contexto geral, como um meta-repositório (repositório de repositórios). Assim, seria possível organizar diferentes tipos de conhecimento, adaptados às necessidades de cada comunidade.

\section{REFERÊNCIAS}

Douglas B Bamforth. 1986. Technological efficiency and tool curation. American antiquity 51, 1 (1986), 38-50.

Clarisse Sieckenius de Souza and Carla Faria Leitão. 2009. Semiotic engineering methods for scientific research in HCI. Synthesis Lectures on Human-Centered Informatics 2, 1 (2009), 1-122.

Clarisse Sieckenius de Souza, Carla Faria Leitão, Raquel Oliveira Prates, and Elton José da Silva. 2006. The semiotic inspection method. In Proceedings of VII Brazilian symposium on Human factors in computing systems. ACM, 148-157.

Marcella Leandro Costa de Souza, Lidia Silva Ferreira, Raquel Oliveira Prates, and Marília Lyra Bergamo. 2015. StoreAnt: A System to Support Finding Collaborative Systems Evaluation Methods. Springer International Publishing, Cham, 482-485. https://doi.org/10.1007/978-3-319-227238_43

Lidia Silva Ferreira and Raquel Oliveira Prates. 2013. RepositóRio Virtual Para Catalogação De MéTodos De Avaliação Para Sistemas Colaborativos. In Proceedings of the X Brazilian Symposium in Collaborative Systems (SBSC '13). Sociedade Brasileira de Computação, Porto Alegre, Brazil, Brazil, Article 152, 4 pages. http://dl.acm.org/citation.cfm?id=2542508. 2542536

Barbara Kitchenham. 2004. Procedures for performing systematic reviews. Keele, UK, Keele University 33, 2004 (2004), 1-26.

Raquel Oliveira Prates and Alberto Barbosa Raposo. 2006. Desafios Para Testes De Usuários Em Sistemas Colaborativos - Lições De Um Estudo De Caso. In Proceedings of VII Brazilian Symposium on Human Factors in Computing Systems (IHC '06). ACM, New York, NY, USA, 9-12. https: //doi.org/10.1145/1298023.1298051

Natália Sales Santos, Lidia Silva Ferreira, and Raquel Oliveira Prates. 2012. Caracterização das Adaptações em Métodos de Avaliação para Aplicações Colaborativas. In Proceedings of the 11th Brazilian Symposium on Human Factors in Computing Systems (IHC '12). Brazilian Computer Society, Porto Alegre, Brazil, Brazil, 297-300. http://dl.acm.org/citation.cfm?id= 2393536.2393579

Elena Voyloshnikova and Margaret-Anne Storey. 2014. Towards understanding digital information discovery and curation. In Proceedings of 24th Annual International Conference on Computer Science and Software Engineering. IBM Corp., 247-261.

Yu Wu, Jessica Kropczynski, Raquel Prates, and John Carroll. 2018. Understanding How GitHub Supports Curation Repositories. Future Internet 10, 3 (2018), 29.

Yu Wu, Na Wang, Jessica Kropczynski, and John M Carroll. 2017. The appropriation of GitHub for curation. Peerf Preprints 5 (2017), e2952v1.

Alexey Zagalsky, Carlos Gómez Teshima, Daniel M German, MargaretAnne Storey, and Germán Poo-Caamaño. 2016. How the R community creates and curates knowledge: a comparative study of stack overflow and mailing lists. In Proceedings of the 13th International Conference on Mining Software Repositories. ACM, 441-451. 